percentage. It has been noted from the previous study, the prevalence of LBW in BPKIHS is $17.56 \%$. The low birth weight is the leading cause of morbidity and mortality among neonates and one of important parameter known to predispose the newborn to increase morbidity and mortality with LBW due to low hemoglobin level of mother. Hence we designed Cross Sectional descriptive study to find out the association between maternal hemoglobin level with gestational age and birth weight of the babies.

Methods 496 mothers admitted for delivery were recruited and their hemoglobin was measured. Hemoglobin in previous trimesters was noted from their antenatal record. The gestational age and birth weight of the babies recorded. Descriptive statistics was used for baseline characteristics. Comparison of means was done using student's t test and analysis of variance to determine associations between the maternal hemoglobin status and the birth weights.

Results Out of 496, around $38.5 \%$ mother were anemic at some point of time during pregnancy. $42 \%$ were anemic during first trimester, $26.4 \%$ during second and $21.1 \%$ during third trimester. Mean birth weight of babies born to anemic and non-anemic mother was 2876 and 2949 gram respectively $(\mathrm{p}=0.172)$. Mean POG in anemic and non-anemic mother was $392 / 7$ week and $395 / 7$ week respectively $(p=0.337)$. There was $9.28 \%$ premature and $90.72 \%$ mature baby. Frequency of LBW babies in anemic and non-anemic mother were $18.1 \%$ and $15.5 \%$ respectively $(\mathrm{p}=0.58)$. The correlation coefficient of gestational age and birth weight of baby with maternal hemoglobin were $0.003(\mathrm{p}=.975), \quad 0.072(\mathrm{p}=.215), \quad 0.13(\mathrm{p}=0.005)$ and $0.065(\mathrm{p}=0.421), 0.157(\mathrm{p}=0.007), 0.091(\mathrm{p}=0.05)$ in first, second and third trimesters respectively.

Conclusion There were significant positive association between maternal hemoglobin level and birth weight and gestational age of the babies. Birth weight and Gestational age of the babies were low in mothers with anemia and increased as maternal hemoglobin increased and a U-shaped relation was observed between different range of hemoglobin and Period of gestation.

\section{P447 ROLE OF SERUM PROCALCITONIN AS MARKER OF NEONATAL SEPSIS}

'Pradeep Gupta*, ${ }^{2}$ Anil Narang. 'Norvic international hospital, Kathmandu, Nepal;

${ }^{2}$ Chaitanya Hospital, Chandigarh, India

\subsection{6/archdischild-2019-epa.783}

Background Despite the advances in perinatal and neonatal care and use of newer potent antibiotics, the incidence of neonatal sepsis remains high and the outcome is still severe. Early diagnosis of neonatal sepsis followed by appropriate treatment decreases mortality and morbidity in infants.

Objective To study the ROLE OF SERUM PROCALCITONIN AS A MARKER OF NEONATAL SEPSIS and To compare procalcitonin with CRP as a diagnostic marker for neonatal sepsis

Methodology Hospital Based prospective observational study.

50 neonates (preterm \&term) with clinically suspected sepsis were studied during 1 year from Jan 2016 to Dec 2016 in Chaitanaya Hospital Chandigarh. Conventional sepsis workup was done in all cases and the diagnosis of neonatal sepsis was proved based on the results of blood culture. The serum Procalcitonin was measured by quantitative Enzyme linked iimunofluroscence assay and the results were compared to CRP levels between the neonates with or without proven sepsis.

Results Of the total 220 babies admitted in NICU during that period 50 were eligible for study and analyzed.. 24\% babies had Definite Sepsis, 60\% had Probable Sepsis and 16\% babies had No Sepsis. Of the neonates with suspected sepsis $24 \%$ had culture positive and $76 \%$ were culture negative. Mean PCT level was $13.27+-33.2 \mathrm{ng} / \mathrm{ml}$. The mean PCT levels were higher in Meningitis group (Mean PCT-26.45) than no meningitis group.(p value-0.216).The mean PCT levels was highest in neonates whose TLC $>5000$ (Mean PCT-18.5) (p value-0.002). The mean PCT levels were higher in all 3 infection groups in neonates with $\mathrm{CRP}>0.5 \mathrm{mg} / \mathrm{dl}$ (positive) than that of neonates with $\mathrm{CRP} \leq 0.5 \mathrm{mg} / \mathrm{dl}$ (negative). Mean PCT levels were $0.433,52.22$ and 27.95 in no infection, probable infection and definite infection group respectively. ( $\mathrm{p}$ value0.001) Evaluating CRP as a diagnostic marker for definite neonatal sepsis with cut off value as $0.5 \mathrm{mg} / \mathrm{dl}$, had sensitivity of $41.67 \%$, Specificity of $89.47 \%$, Positive Predictive Value of $55.56 \%$ and Negative Predictive value of $82.93 \%$.Evaluating PCT as a diagnostic marker for definite neonatal sepsis. The Sensitivity, Specificity, Positive Predictive Value, Negative Predictive Value were $83.3 \%, 26.32 \%, 26.32 \%$ and $83.3 \%$ respectively taking cut- off level of procalcitonin to be $>0.5$ $\mathrm{ng} / \mathrm{ml}$.

Conclusion The importance of procalcitonin in diagnosing neonatal septicaemia becomes more useful when it is used along with other investigations. Especially in identifying the group of neonates who may not be infected and may not require antibiotics.

\section{P448 9 YEAR EXPERIENCE AND OUTCOMES OF TRUNCUS ARTERIOSUS IN A TERTIARY NEONATAL INTENSIVE CARE UNIT}

Elizabeth Louise Stockley*, Tarak Desai, Shree Vishna Rasiah. Birmingham Women's and Children's NHS Foundation Trust, Birmingham, UK

10.1136/archdischild-2019-epa.784

Background Congenital heart defect affects around 10 in a 1000 live born babies. Truncus arteriosus is a rare conotruncal defect in which a common arterial trunk supplies systemic, pulmonary and coronary circulation. The incidence of truncus arteriosus is quoted as $1 \%$ of all congenital heart malformations.

Aim To evaluate the initial neonatal management and longterm outcomes of newborns with truncus arteriosus.

Method We conducted a retrospective review of all newborns with truncus arteriosus admitted to our neonatal unit and referred to a surgical centre between 2010 and 2018. Data were collected from Badger and Cardiology databases to evaluate the initial neonatal management and long-term outcomes of this cohort of patients.

Results Fifteen newborns with truncus arteriosus were admitted to our neonatal unit. Their mean gestational age was 36 weeks with a mean birth weight of 2407 grams. Cardiac defects were detected prenatally in 12 patients (80\%). Three babies were diagnosed postnatally. One following failed pulse oximetry screening requiring non-invasive respiratory support and 2 diagnosed following on-going respiratory support on echocardiography. Respiratory support was initiated within the 